\title{
Deeplmpute: an accurate, fast, and scalable deep neural network method to impute single-cell RNA-seq data
}

\author{
Cédric Arisdakessian ${ }^{1}$, Olivier Poirion ${ }^{2}$, Breck Yunits ${ }^{2}$, Xun Zhu ${ }^{2,3}$ and Lana X. Garmire ${ }^{4^{*}}$ (D)
}

\begin{abstract}
Single-cell RNA sequencing (scRNA-seq) offers new opportunities to study gene expression of tens of thousands of single cells simultaneously. We present Deeplmpute, a deep neural network-based imputation algorithm that uses dropout layers and loss functions to learn patterns in the data, allowing for accurate imputation. Overall, Deeplmpute yields better accuracy than other six publicly available scRNA-seq imputation methods on experimental data, as measured by the mean squared error or Pearson's correlation coefficient. Deeplmpute is an accurate, fast, and scalable imputation tool that is suited to handle the ever-increasing volume of scRNA-seq data, and is freely available at https://github.com/lanagarmire/Deeplmpute.
\end{abstract}

Keywords: RNA-seq, Single-cell, Imputation, Deep learning, Machine learning, Neural network, Dropout, Deeplmpute

\section{Introduction}

The RNA sequencing technologies keep evolving and offering new insights to understand biological systems. In particular, single-cell RNA sequencing (scRNA-seq) represents a major breakthrough in this field. It brings a new dimension to RNA-seq studies by zooming in to the single-cell level. Currently, various scRNA-seq platforms are available such as Fluidigm- and Drop-Seq-based methods. While Drop-Seq can process thousands of cells in a single run, Fluidigm generally processes fewer cells but with a higher coverage. In particular, 10X Genomics' platform is gaining popularity in the scRNA-seq community due to its high yield and low cost per cell. Consequently, an increasing amount of studies have taken advantage of these technologies to discover new cell types [1, 2], new markers for specific cell types $[1,3,4]$, and cellular heterogeneity [4-9].

Despite these advantages, scRNA-seq data are very noisy and incomplete [10-12] due to the low starting amount of mRNA copies per cell. Datasets with more than $70 \%$ missing (zero) values are frequently observed in an scRNA-seq experiment. These apparent zero

\footnotetext{
* Correspondence: Igarmire@med.umich.edu

${ }^{4}$ Department of Computational Medicine and Bioinformatics, University of Michigan, Ann Arbor, MI 48105, USA

Full list of author information is available at the end of the article
}

values could be truly zeros or false negatives. The latter phenomenon is called "dropout" [13] and is due to failure of amplification of the original RNA transcripts. Among genes of various lengths, shorter genes were more likely to be dropped out [14]. Such bias may increase further during the subsequent amplification steps. As a result, dropout can affect downstream bioinformatics analysis significantly, such as clustering [15] and pseudo-time reconstruction [16], as it decreases the power of the studies and introduces biases in gene expression. To correct such issue, analysis platforms such as Granatum [17] have included an imputation step, in order to improve the downstream analysis.

Currently, several imputation algorithms have been proposed, based on different principles and models. MAGIC [18] focuses on cell/cell interactions to build a Markov transition matrix and smooth the data. ScImpute [19] builds a LASSO regression model for each cell and imputes them iteratively. SAVER [20] is a Bayesianbased model using various prior probability functions. DrImpute [21] is a clustering-based method and uses a consensus strategy: it estimates a value with several cluster priors or distance matrices and then imputes by aggregation. VIPER is a recent published statistical method that looks at cell/cell interaction to fit a linear model for each cell. Instead of using a LASSO regression as for 
scImpute, the authors use a hard thresholding approach to limit the number of predictors [22]. Most recently, DCA builds an auto-encoder to model the genes distribution using a zero inflated negative binomial prior. To this end, the auto-encoder tries to predict the genes' mean, standard deviation, and dropout probability [23]. As the low quality of the scRNA-seq datasets continues to be a bottleneck while the measurable cell counts keep increasing, the demand for faster and scalable imputation methods also keeps increasing [23-25]. While some of these earlier algorithms may improve the quality of original datasets and preserve the underlying biological variance [26], most of these methods demand extensive running time, impeding their adoption in the ever-increasing scRNA-seq data space.

Here, we present a novel algorithm, DeepImpute, as the next generation imputation method for scRNA-seq data. DeepImpute is short for "Deep neural network Imputation". As reflected by the name, it belongs to the class of deep neural-network models [27-29]. Recent years, deep neural network algorithms have gained much interest in the biomedical field [30], ranging from applications from extracting stable gene expression signatures in large sets of public data [31] to stratify phenotypes [32] or impute missing values [33] using electronic health record (EHR) data. In this report, we construct DeepImpute models by splitting the genes into subsets and build sub-networks to increase its efficacy and efficiency. Using accuracy metrics, we demonstrate that DeepImpute performs better than the six other recently published imputation methods mentioned above (MAGIC, DrImpute, ScImpute, SAVER, VIPER, and DCA). It also improved the downstream analysis results, on clustering using both real and simulated datasets, as well as on differential expression using a simulated dataset. We additionally show the superiority of DeepImpute over the other methods in terms of computational running time and memory use. Moreover, DeepImpute allows to train the model with a subset of data to save computing time, with little sacrifice on the prediction accuracy. In summary, DeepImpute is a fast, scalable, and accurate next generation imputation method capable of handling the ever-increasing scRNA-seq data.

\section{Results}

\section{Overview of the Deeplmpute algorithm}

DeepImpute is a deep neural network model that imputes genes in a divide-and-conquer approach, by constructing multiple sub-neural networks (Additional file 1: Figure S1). Doing so offers the advantage of reducing the complexity by learning smaller problems and fine-tuning the subneural networks [34]. For each dataset, we select to impute a list of genes, which have a certain variance over mean ratio (default $=0.5$ ). Each sub-neural network aims to understand the relationship between the input genes (input layer) and a subset of target genes (output layer) (Fig. 1). Users can set the size of the target genes, and we set 512 as the default value, as it offers a good trade-off between speed and stability. As shown in Fig. 1, each sub-neural network is composed of four layers. The input layer consists of genes that are highly correlated with the target genes, followed by a 256-neuron dense hidden layer, a dropout layer with $20 \%$ dropout rate (note: not the dropout rate in the single cell data matrix) of neurons which avoid overfitting (Additional file 2: Figure S1), and the output neurons made of the abovementioned target genes. We use rectified linear unit (ReLU) as the default activation function and train each sub-model in parallel by splitting the data to train (95\% of the cells) and test (5\%) data. We stop the training if the test loss does not improve for 5 consecutive epochs or the number of epochs exceeds 500, whichever is smaller. Because of the simplicity of each sub-network, we observe very low variability due to hyperparameter tuning. As a result, we set the default parameters for batch size at 64 and learning rate at 0.0001 . Further information about the network parameters are described in the "Methods" section. In the following sections, we describe the comprehensive evaluations of DeepImpute.

\section{Deeplmpute is the most accurate among imputation methods on scRNA-seq data}

We tested the accuracy of imputation on four publicly available scRNA-seq datasets (Additional file 3: Table S1): two cell lines, Jurkat and 293T (10X Genomic); one mouse neuron cells dataset (10X Genomics); and one mouse interfollicular epidermis dataset deposited in GSE67602. We compared DeepImpute with six other state-of-the-art, representative algorithms: MAGIC, DrImpute, ScImpute, SAVER, VIPER, and DCA. Since the real dropout values are unknown, we evaluated the different methods by randomly masking (replacing with zeros) a part of the expression matrix of a scRNA-seq dataset (Additional file 1: Figure S2) and then measure the differences between the inferred and actual values of the masked data. In order to mimic a more realistic dropout distribution, we estimated the masking probability function from the data (see the "Methods" section). We measured the accuracies using the two metrics on the masked values: Pearson's correlation coefficient and mean squared error (MSE), as done earlier [20,35].

Figure 2 shows all the results of imputation accuracy metrics on the masked data. DeepImpute successfully recovers dropout values from all ranges, introduces the least distortions and biases to the masked values, and yields both the highest Pearson's correlation coefficient and the best (lowest) MSE in all datasets (Fig. 2a and c). DCA, another neuron-network-based method, has the second best performance after DeepImpute, based on 


\section{Sub-neural network for each sub scRNA-Seq set (default 512 genes)}

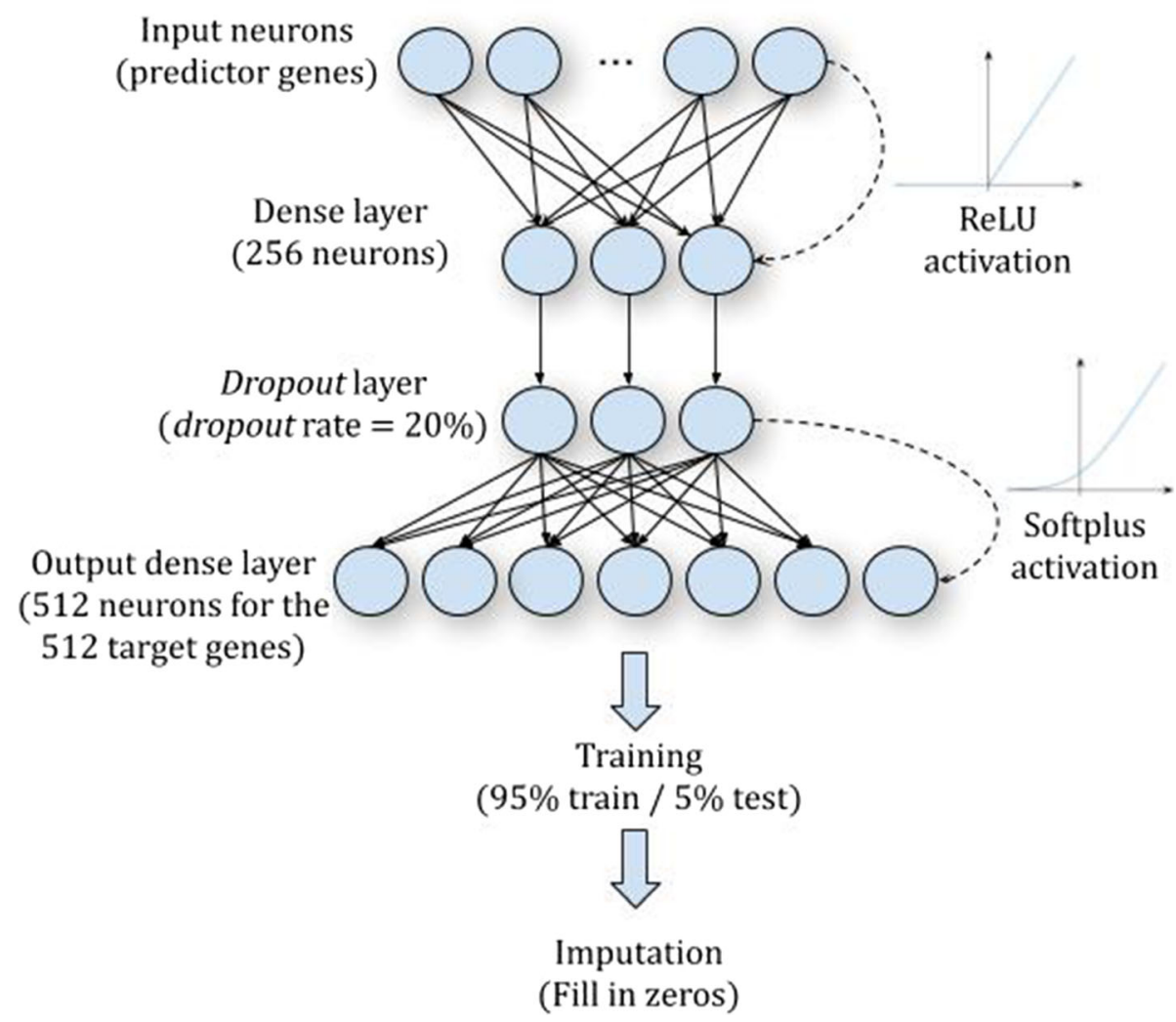

Fig. 1 (Sub) Neural network architecture of Deeplmpute. Each sub-neural network is composed of four layers. The input layer is genes that are highly correlated with the target genes in the output layer. It is followed by a dense hidden layer of 256 neurons dense layer and a dropout layer (dropout rate $=20 \%$ ). The output layer consists of a subset of target genes (default $N=512$ ), whose zero values are to be imputed

both MSE and Pearson's correlation coefficient. On the contrary, other methods present various issues: VIPER tends to underestimate the original values, as reflected by the largest MSEs. scImpute has the widest range of variations among imputed data and generates the lowest Pearson's correlations. MAGIC, SAVER, and DrImpute have intermediate performances compared to other methods. However, SAVER persistently underestimate the values, especially among the highly expressed genes. We further examined MSE distributions calculated on the gene and cell levels (Fig. 2b). DeepImpute is the clear winner with consistently the best (lowest) MSEs both at gene and cell levels on all datasets, which are significantly lower than all other imputation methods $(p<$ 0.05). scImpute and VIPER give the two highest MSEs at the cell level, whereas VIPER consistently has the highest MSE at the gene level (Fig. 2b). Other methods are ranked in between, with varying rankings depending on the datasets and gene or cell level. As internal controls, we also compared DeepImpute (with ReLU activation) with 2 variant architectures: the first one with no hidden layers and the second one with the same hidden layers but using linear activation function (instead of ReLU). As shown in Additional file 2: Figure S2, DeepImpute (with ReLU activation) yields Pearson's correlation coefficients and MSEs that are either on par or better than the two other variant architectures. For GSE67602 and neuron9k datasets generated from complex animal primary tissues, DeepImpute (with ReLU activation) performs better; for Jurkat and 293T datasets generated from cell lines, the results are comparable. This suggests that DeepImpute (with ReLU activation) handles complex datasets better than its variants. In summary, DeepImpute yields the highest accuracy in the datasets studied, among the imputation methods in comparison.

DeepImpute improves the gene distribution similarity with FISH experimental data

Another way to assess the imputation efficiency is through experimental validation on scRNA-Seq data. 


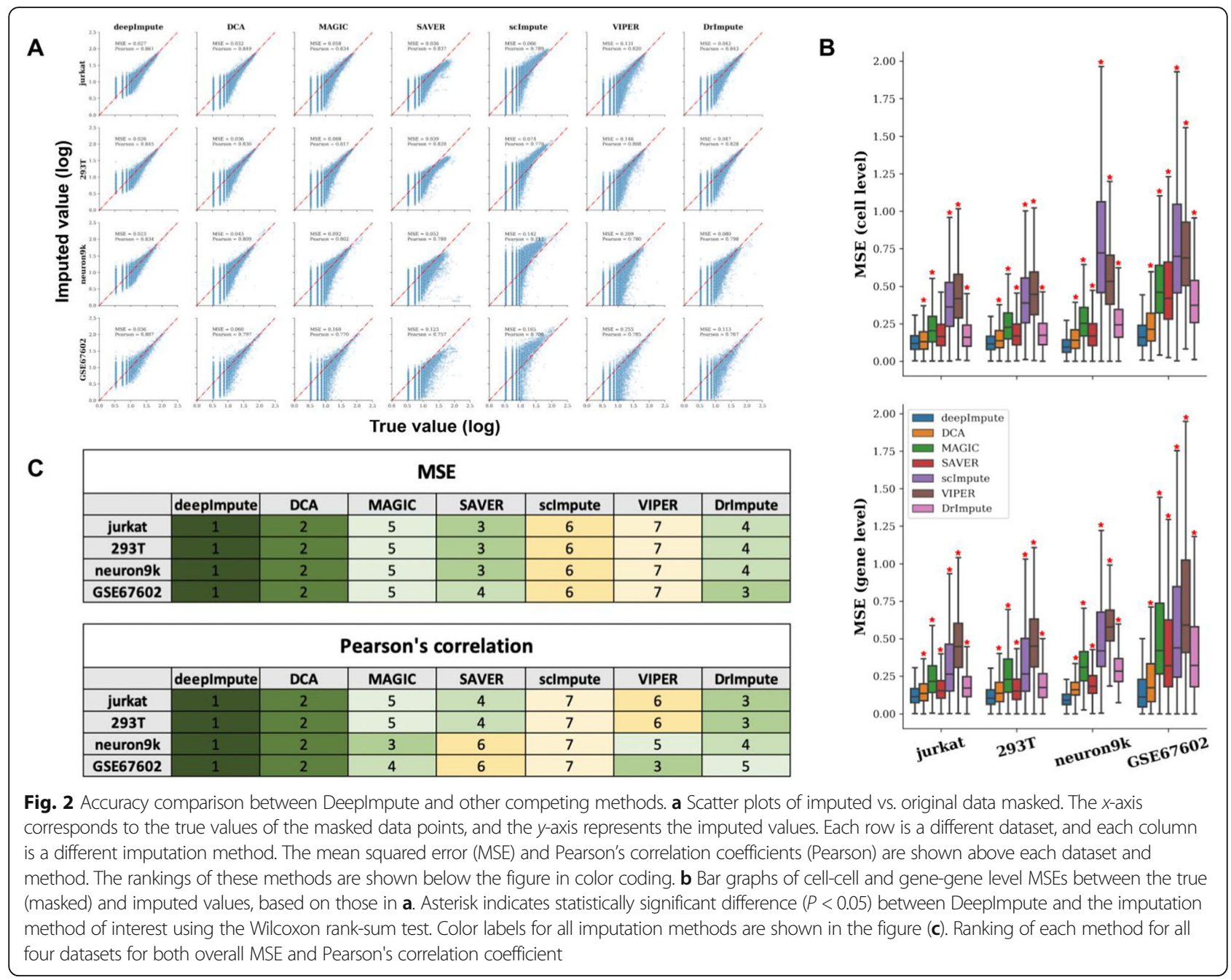

Single-cell RNA FISH is such a method that directly detects a small number of RNA transcripts in a single cell. Torre et al. measured the gene expression of a melanoma cell line using both RNA FISH and Drop-Seq and compared their distribution using their GINI coefficients (see the "Methods" section) [36]. Similarly, we compared the same list of genes using their GINI coefficients of RNA FISH vs. those after imputation (or raw scRNA-seq data). DrImpute could not handle the large cell size and was omitted from comparison. Comparing to Pearson's correlation coefficient between RNA FISH and the raw scRNAseq data $(-0.260)$, three methods, DeepImpute, SAVER, and DCA, have the top 3 most improved and positive correlation coefficients, with values of $0.984,0.782$, and 0.732 , respectively. VIPER barely changed the GINI coefficients, whereas scImpute had a correlation coefficient $(-0.451)$ even lower than the raw scRNA-seq dataset (Fig. 3a). For MSE, all other imputation methods achieved better (smaller) MSEs compared to the raw scRNA-seq results (MSE $=0.281$ ), except VIPER which gives the same MSE as raw data. Echoing the results of correlation coefficient, three methods, SAVER, DeepImpute, and DCA, give the lowest MSEs. DeepImpute is the second most accurate method with an MSE (MSE $=0.0256$ ), closely after SAVER $(\mathrm{MSE}=0.0152)$ and followed by DCA (MSE $=0.0436)$. Additionally, we compared the distributions of each gene before and after various imputation methods, as well as in FISH experiments (Fig. 3b). Overall, DeepImpute (blue curves) yields the most similar distributions to those of FISH experiments (gray curves) for three of five genes (LMNA, MITF, and TXNRD1), with K-S test statistics of $0.08,0.15$, and 0.18 , respectively. For KDM5A, it achieved 2nd best K-S statistics 0.18 , almost the same as DCA (0.17). It does not perform as well for gene VGF (K-S statistic of 0.44 ), which has over $40 \%$ zero values even in RNA-FISH data (56\% in raw Drop-Seq data). Altogether, the FISH validation results clearly show that DeepImpute improves the data quality by imputation.

\section{Deeplmpute improves downstream functional analysis}

Another way to assess possible benefits of imputation is to conduct downstream functional analysis. Towards 


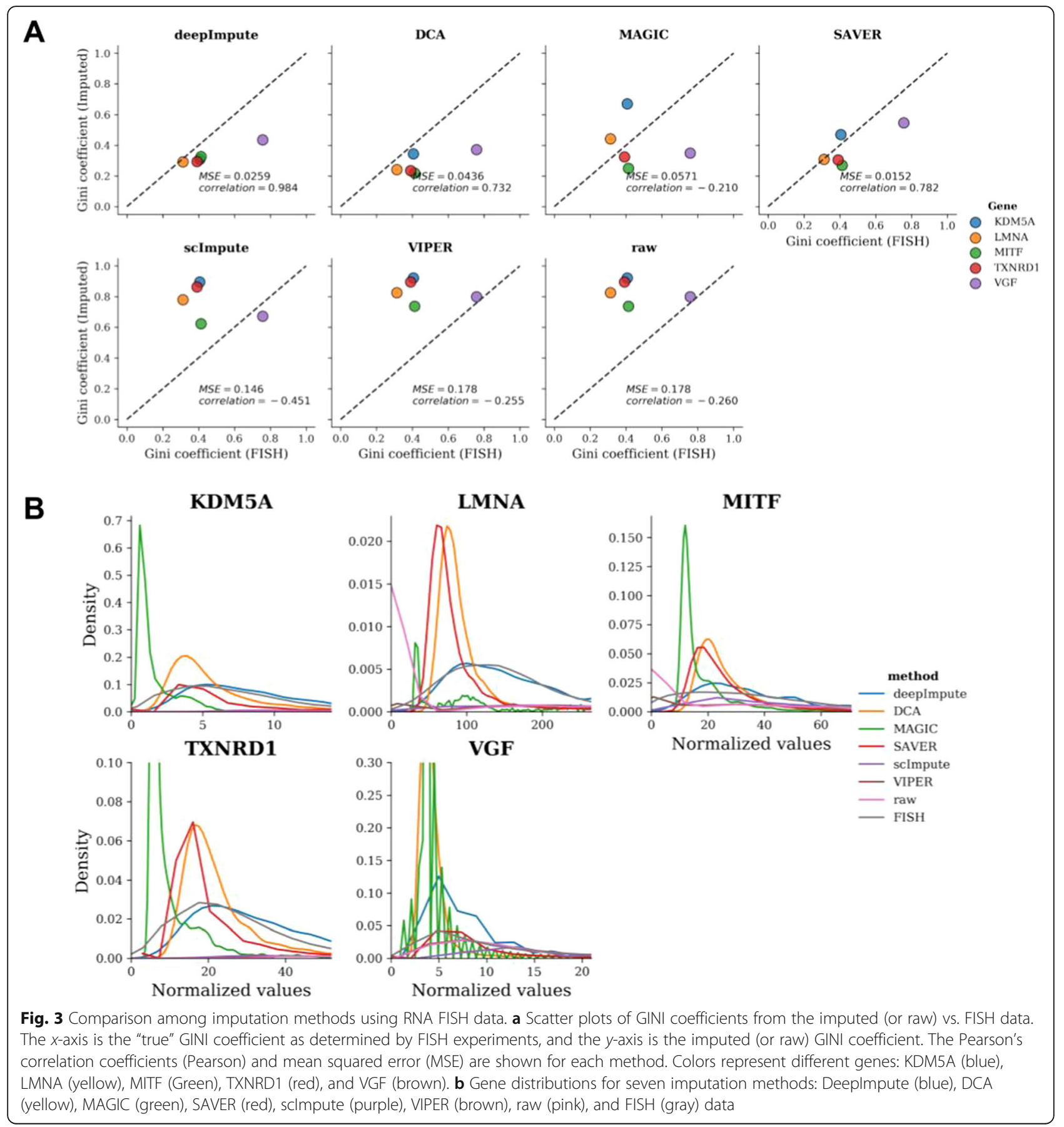

this, we utilized additional experimental and simulation datasets. We use an experimental dataset (Hrvatin) from GSE102827, composed of 48,267 annotated primary visual cortex cells from mice and which had 33 prior cell type labels [37]. Using the Seurat pipeline implemented in Scanpy, we extracted the UMAP [38] components (Fig. 4a). We then performed cell clustering using the Leiden clustering algorithm [39], an improved version of the Louvain algorithm [40]. We measure the accuracy of clustering assignments using various metrics, including the Adjusted Rand Index (ARI), the Adjusted Mutual Score (AMS), the Fowlkes-Mallow Index (FMI), and Silhouette Index (SI) to exam UMAP cluster shapes (Fig. 4b). Due to the size of the Hrvatin dataset, we could not run DrImpute and VIPER (speed issues) as well as scImpute (speed and memory issues), but only DeepImpute, DCA, MAGIC, and SAVER. DeepImpute manages to disentangle many clusters (Fig. 4a), resulting in the most improved clustering metrics compared to the scenario without imputation (Fig. 4b). DCA, the other deep neural-network-based method, also 


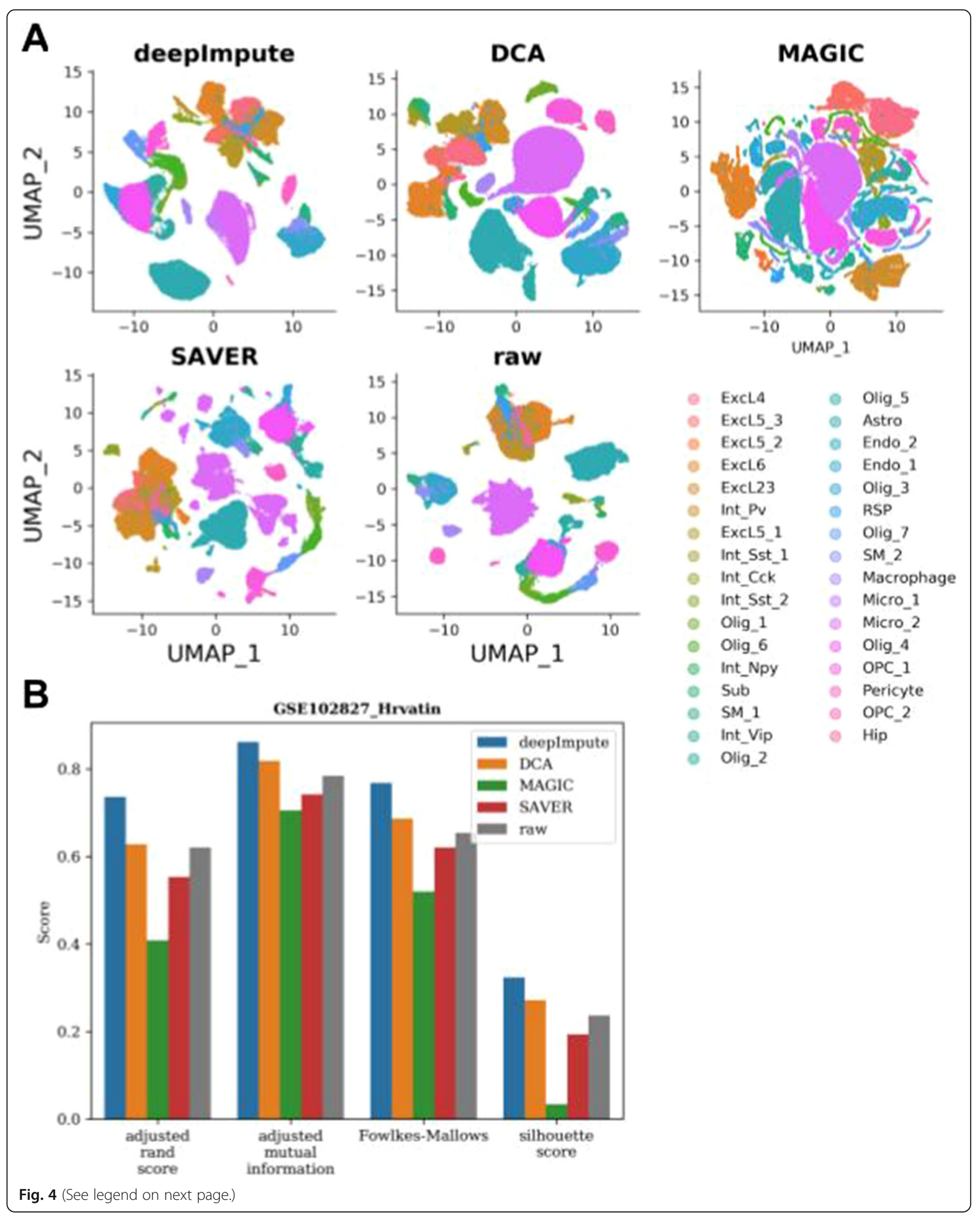


(See figure on previous page.)

Fig. 4 Comparison on effect of imputation on downstream function analysis of the experimental data (GSE102827). a UMAP plots of Deeplmpute, DCA, MAGIC, SAVER, and raw data (scImpute, DrImpute, and VIPER) failed to run due to the large cell size of 48,267 cells). Colors represent original cell type labels as annotated. b Accuracy measurements of clustering using various metrics: adjusted Rand index (adjusted_rand_score), adjusted mutual information (adjusted_mutual_info_score), Fowlkes-Mallows Index (Fowlkes-Mallows), and Silhouette coefficient (Silhouette score). Higher values indicate better clustering accuracy. Bar colors represent different methods: Deeplmpute (blue), DCA (orange), MAGIC (green), SAVER (red), and raw data (brown)

slightly improves the clustering metrics (Fig. 4b). On the contrary, MAGIC and SAVER decrease, rather than improving the clustering outcome. Notable, MAGIC manages to split many cell types but also highly distorts the data (Fig. 4a). SAVER disentangles some clusters, but also splits some clusters beyond the original cell type labels (Fig. 4a).

Given lack of absolute truth of class labels in experimental data, we next generated a simulation data using Splatter. This simulation dataset (sim) is composed of 4000 genes and 2000 cells, which are split into 5 cell types (proportions: 5\%/5\%/10\%/20\%/20\%/40\%). DeepImpute successfully separates cell types on the simulation, closely followed by scImpute (Fig. 5a). These observations are confirmed by the evaluation metrics, where DeepImpute achieves almost perfect scores for ARI, AMS, and FMI and significantly increases the Silhouette score compared to the raw data (Fig. 5b). Next, we compare all seven imputation methods for their capabilities to recover differentially expressed genes in the simulation data (Fig. 5c). For each method, we extracted the top 500 differentially expressed genes in each cell type and compared with the true differentially expressed genes. Overall, DeepImpute has the highest precision $(\mathrm{AUC}=0.893)$ at detecting differentially expressed genes, compared to those of no imputation and other imputation methods. All together, these results from both experimental and simulation data show unanimously that DeepImpute improves downstream functional analysis.

\section{Deeplmpute is a fast and memory efficient package}

As scRNA-seq becomes more popular and the number of sequenced cells scales exponentially, imputation methods will have to be computationally efficient to be widely adopted. With such a goal in mind, we choose the Mouse1M dataset to evaluate the computational speed and memory usage among different imputation methods. We use Mouse1M dataset as it has the highest number of cells to assess how adaptive each method is.

We downsampled the Mouse1M data, ranging in size from 100 to $50 \mathrm{k}$ cells $(100,500,1 \mathrm{k}, 5 \mathrm{k}, 10 \mathrm{k}, 30 \mathrm{k}, 50 \mathrm{k})$. We ran the imputations three times and measured the runtime (for both training and testing steps) and memory load on an 8-core machine with $30 \mathrm{~GB}$ of memory. DeepImpute, DCA, and MAGIC outperformed the other four packages on speed (Fig. 6a), and DeepImpute is the most advantageous when the cell counts get large ( $>30 \mathrm{k})$.
DCA is consistently and slightly slower than DeepImpute through all tests. The other four imputation methods (scImpute, DrImpute, VIPER, and SAVER) are significantly slower and consume significantly more memory (Fig. 6b). The slow computation time of VIPER and DrImpute are due to lack of parallelization. VIPER is unable to scale beyond $5 \mathrm{k}$ cells within $24 \mathrm{~h}$, while scImpute exceeded the $30 \mathrm{~GB}$ of memory available and failed to run on more than 10k cells. For memory, DeepImpute and DCA, two neural-network-based methods, are the most efficient, and their merits are much more pronounced on large datasets (Fig. 6b). MAGIC uses a similar amount of memory as DeepImpute and DCA on smaller datasets; however, as the dataset size increases beyond 10k cells, it requires significantly more memory. It hits an out of memory error and is unable to finish the 50k cell imputation on our 30GB machine. In all, judging by both computation speed and memory efficiency on larger datasets, DeepImpute and DCA tops the other five methods.

\section{Deeplmpute is a scalable machine learning method}

Unlike the other imputation methods, DeepImpute first fits a predictive model and then performs imputation separately. The model fitting step uses most of the computational resources and time, while the prediction step is very fast. We then asked the question what is the minimal fraction of the dataset needed to train DeepImpute and obtain efficient imputation without extensive training time. Hence, we used the neuron9k dataset and evaluated the effect of different subsampling fraction $(5 \%$, $10 \%, 20 \%, 40 \%, 60 \%, 80 \%, 90 \%, 100 \%)$ in the training phase on the imputation prediction phase. We randomly picked a subset of the samples for the training step and computed the accuracy metrics (MSE, Pearson's correlation coefficient) on the whole dataset, with 10 repetitions under each condition. Model performance improvement begins to slow down at around $40 \%$ of the cells (Fig. 6c). Specifically, from 40 to $100 \%$ fraction of data as the training set, the MSE decreases slightly from 0.121 to 0.116 , and Pearson's coefficient score marginally improves from 0.880 to 0.884 . These experiments demonstrate another advantage of DeepImpute over the other competing methods, that is, the use of only a fraction of the data set will reduce the running time even more with little sacrifice to the accuracy of the imputed results. 


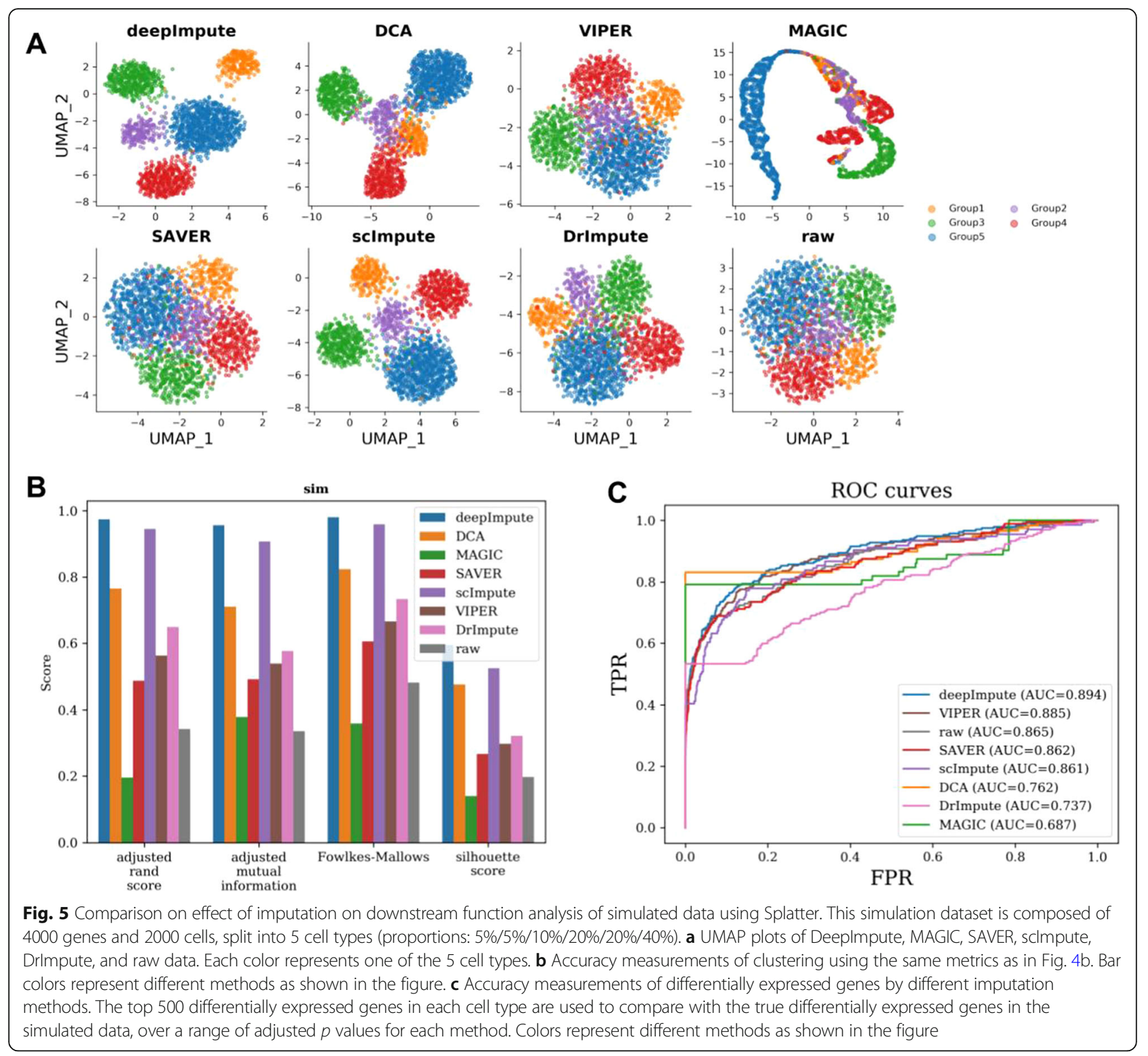

\section{Discussion}

Dropout values in scRNA-seq experiments represent a serious issue for bioinformatic analyses, as most bioinformatics tools have difficulty handling sparse matrices. In this study, we present DeepImpute, a new algorithm that uses deep neural networks to impute dropout values in scRNA-seq data. We show that DeepImpute not only has the highest overall accuracy using various metrics and a wide range of validation approaches, but also offers faster computation time with less demand on the computer memory. In both simulated and experimental datasets, DeepImpute shows benefits in increasing clustering results and identifying significantly differentially expressed genes, even when other imputation methods are not desirable. Furthermore, it is a very "resilient" method. The model trained on a fraction of the input data can still yield decent predictions, which can further reduce the running time. Together, these results demonstrate consistently and robustly that DeepImpute is an accurate and highly efficient method, and it is likely to withstand the tests of time, given the rapid growth of scRNA-Seq data volume.

Through systematic comparisons, two deep-learningbased methods, DeepImpute and DCA, show overall advantages over other methods, between which DeepImpute performs even better. Several unique properties of DeepImpute contribute to its superior performance. One of them is using a divide-and-conquer approach. This approach has several benefits. First, contrary to an autoencoder as implemented in DCA, the subnetworks are 


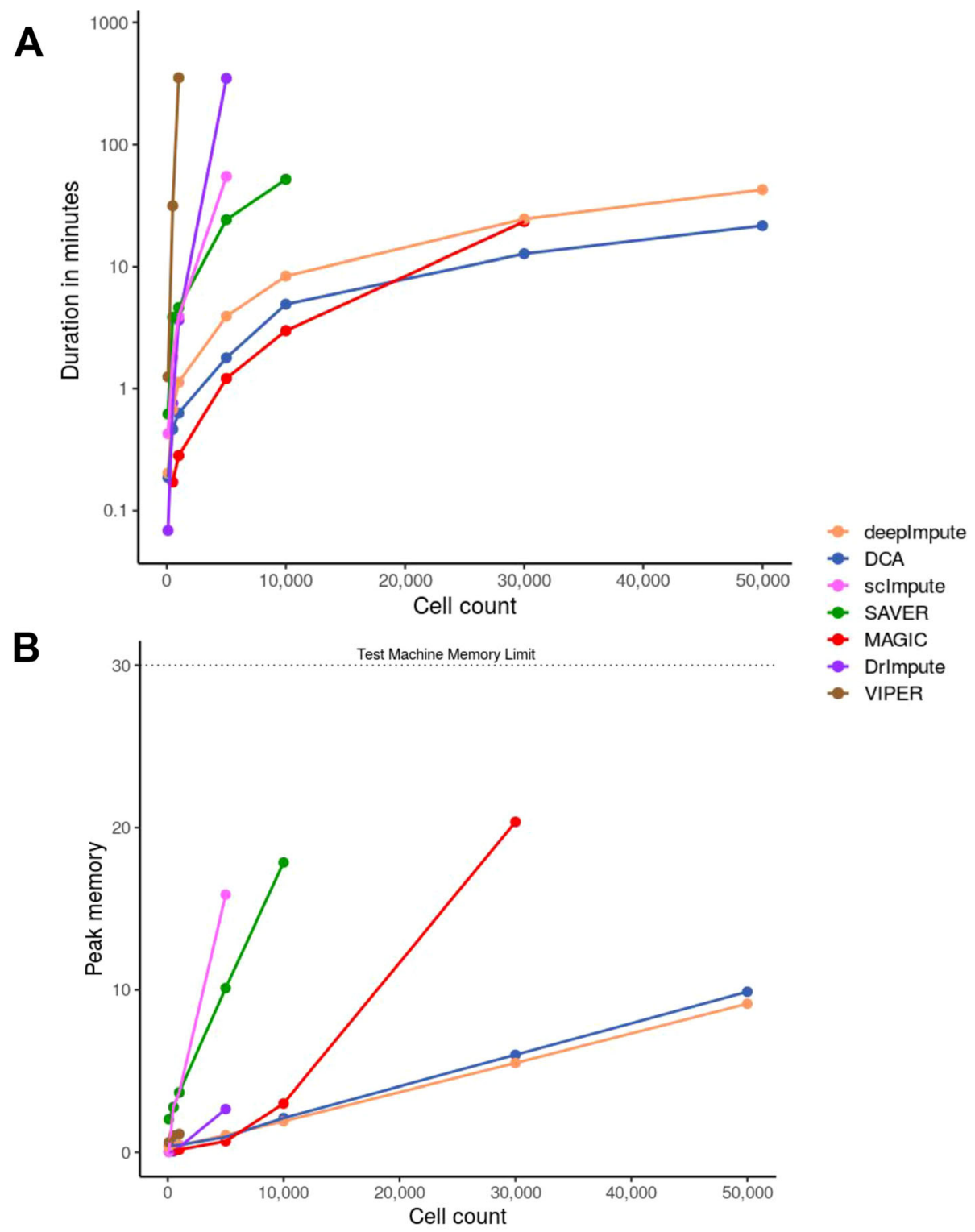

C

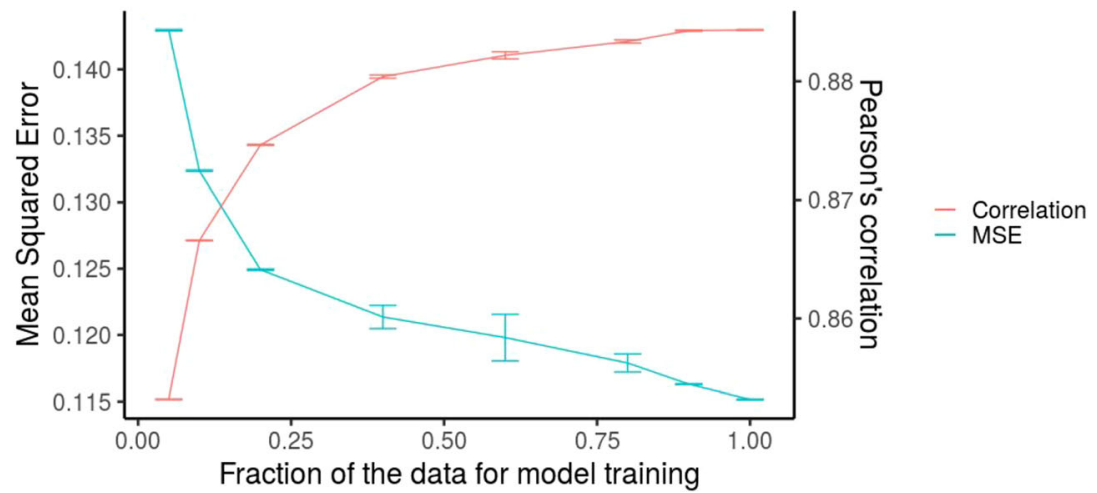

Fig. 6 (See legend on next page.) 


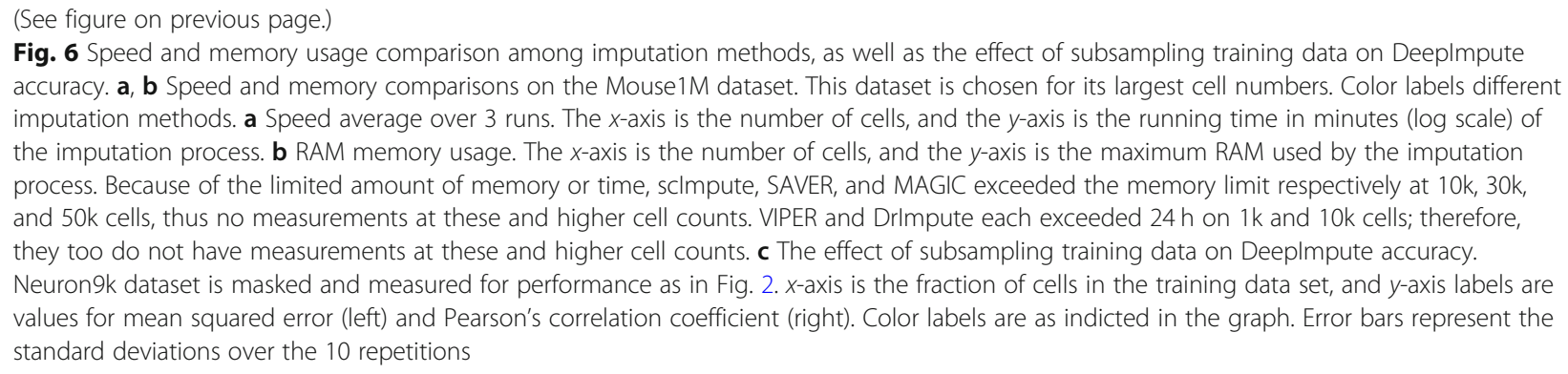

trained without using the target genes as the input. It reduces overfitting while enforcing the network to understand true relationships between genes. Second, splitting the genes into subsets results in a lower complexity in each sub-model and stabilizing neural networks. As a result, a small change in the hyperparameters has little effect on the result. Using a single set of hyperparameters, DeepImpute achieves the highest accuracies in all four experimental datasets (Fig. 2a). Third, splitting the training into sub-networks results in increased speed as there are fewer input variables in each subnetwork. Also, training of each sub-network is done in parallel on different threads, which is more difficult to do with one single neural network.

Unlike some other imputation algorithms in comparison, DeepImpute is a machine learning method. The training and the prediction processes of DeepImpute are separate, and this may provide more flexibility when handling large datasets. Moreover, we have shown that using only a fraction of the overall samples, one can still obtain decent imputation results without sacrificing the accuracy of the model much, thus further reducing the running time. Perhaps, another advantage of DeepImpute over other methods is that one can pre-train a dataset of a cell type (or cell state) on another cell type (or cell state) decently. This pre-training process is very valuable in some cases, such as when the number of cells in the dataset is too small to construct a high-quality model. Pre-training can also largely reduce the overall computation time, since DeepImpute spends most of the time on training the samples. Thus, it is also a good strategy when the new, large dataset is very similar to the dataset used in pre-training.

An enduring imputation method has to adapt to the ever-increasing volume of scRNA-seq data. DeepImpute is such a method, implemented in deep learning framework where new solutions for speed improvements keep appearing. One example is the development of neural network-specific hardware (such as tensor processing units [41], or TPUs) which are now available on Google Cloud. TPU can dramatically accelerate the tensor operations and thus the imputation process. We were already able to deploy DeepImpute in a Google Cloud environment where TPUs are already available. Another example is the development of frameworks that efficiently use computer clusters to parallelize tasks such as Apache-Spark [42] or Dask [43]. Such resources will help DeepImpute and similar deep-learning methods, such as scDeepCluster designed for clustering analysis [44], achieve even higher peed over time and keep up with the development of scRNA-seq technologies.

\section{Methods}

\section{The workflow of Deeplmpute}

DeepImpute is a deep neural network-based imputation workflow, implemented with the Keras [45] framework and TensorFlow [46] in the backend. Below, we describe the workflow in four steps: preprocessing, architecture, training procedure, and imputation.

\section{Preprocessing}

The first step of DeepImpute is selecting the genes for imputation, based on the variance over mean ratio (default $=0.5$ ), which are deemed interesting for downstream analyses [47, 48]. For efficiency, we adopt a divide-and-conquer strategy in our deep learning imputation process. We split the genes into $N$ random subsets, each with $S$ numbers of genes, which we call "target genes." By default, $S$ is set as 512 . If the number of target genes is not a multiple of this number, we round the number of genes to impute in $N+1$ subsets of deep neural networks. The details of this step are illustrated in Additional file 1: Figure S1.

\section{Network architecture}

For each subset, we train a neural network of four layers: the input layer of genes that are correlated to the target genes, a 256-neuron fully connected hidden layer with a rectified linear unit (ReLU) activation function, a dropout layer (note: different from dropout data in scRNASeq), and an output layer of $S$ target genes. A gene is selected to the input layer, if it satisfies these conditions: (1) it is not one of the target genes and (2) it has top 5 ranked Pearson's correlation coefficient with a target gene. The dropout layer is included after the hidden layer, as a common strategy to prevent overfitting [49]. 
We optimized the dropout rate as $20 \%$, after experimenting the dropout rates from 0 to $90 \%$ (Additional file 2: Figure S1). The other default parameters of the networks include a learning rate of 0.0001 , a batch size of 64 , and a subset size of 512. As internal controls, we also experimented two alternative setups for DeepImpute: one with the same architecture but linear activation function and the other one without hidden layers of neurons.

\section{Training procedure}

The training starts by splitting the cells between a training (95\%) and a test set (5\%). The test set is used at each epoch to measure overfitting. We use a weighted mean squared error (MSE) loss function that gives higher weights to genes with higher expression values. This emphasizes accuracy on high confidence values and avoids over penalizing genes with extremely low values (e.g., zeros). For a given cell c, the loss is calculated as follows:

$$
\text { Loss_c }=\sum Y_{i} \cdot\left(Y_{i}-\hat{Y}_{i}\right)^{2}
$$

where $Y_{i}$ is the value of gene $i$ for cell $\mathrm{c}$ and $\hat{Y}_{i}$ is the corresponding estimated value at a given epoch. For the gradient descent algorithm, we choose an adaptive learning rate method, the Adam optimizer [50], since it is known to perform very efficiently over sparse data [51]. The training stops if it reaches 500 epochs or if the training does not improve for 10 epochs.

\section{Imputation}

Once the network weights are properly trained, we impute the data by filling zeros in the original matrix with the imputed values.

\section{Evaluation metrics}

\section{Accuracy comparison on real datasets}

To evaluate the accuracy of imputation, we apply a random mask to the real single-cell datasets. The masking probability function is estimated in a similar fashion as in splatter [14]. For each gene, we extract the proportion of zeros vs. the mean of those positive values. As done in Splatter, we fit a logistic function to these data points. Next, for each gene in the dataset, we mask ten cells at random using a multinomial distribution: each cell $c_{1} \ldots$ $c_{n}$ has a dropout probability $p_{1} \ldots p_{n}$ given by the logistic function previously fitted. The masked cells are sampled from a multinomial distribution with parameters $\left(q_{1}, q_{2}\right.$, $\left.\ldots, q_{n}\right)$, where $q_{i}=p_{i} / \sum_{i} p_{i} \quad$ are the normalized probability such that $\sum_{i} q_{i}=1$.

These original values are used as "truth values" to evaluate the performance of imputation methods. We used two types of performance metrics: the overall Pearson correlation coefficient and MSE, both on log transformed counts. When needed, we also computed MSE between cells $c_{j}$ and between genes $g_{i}$. Speed and memory comparison: we run comparisons on a dedicated 8core, 30-GB RAM, 100-GB HDD, Intel Skylake machine running Debian 9.4. We record process memory usage at 60-s intervals. For testing data, we use the Mouse1M dataset since it has the largest number of single cells (Additional file 3: Table S1). We filter out genes that are expressed in less than $20 \%$ of cells, leaving 3205 genes in our sample. From this dataset, we generate 7 subsets ranging in size $(100,500,1 \mathrm{k}, 5 \mathrm{k}, 10 \mathrm{k}, 30 \mathrm{k}, 50 \mathrm{k}$ cells). We run each package 3 times per subset to estimate the average computation time. Some packages (VIPER, DrImpute, SAVER, scImpute, and MAGIC) are not able to successfully handle the larger files either due to outof-memory errors (OOM) or exceedingly long run times (> 24h).

\section{Downstream functional analysis Clustering}

We perform cell clustering using the Seurat pipeline implemented in Scanpy. After preprocessing the data, we extract the UMAP components [38] and cluster the cells using the Leiden algorithm recommended in the Scanpy documentation. To assess the performance of the clusters, we use four metrics. For all of them, a value of 1 indicates a perfect clustering, while 0 corresponds to random assignments.

\section{Adjusted mutual information [16]}

It is an entropy-based metric that calculates the shared entropy between two clustering assignments, and is adjusted for chance. The mutual information is calculated by $M I(C, K)=\sum_{i \in C} \sum_{j \in K} P(i, j) \cdot \log \left(\frac{P(i, j)}{P(i) P(j)}\right)$, where $P(i, j)$ is the probability of cell $i$ belonging to both cluster $\mathrm{C}$ and $\mathrm{K}$.

\section{Adjusted Rand index [16]}

It is the ratio of all cell pairs that are either correctly assigned together or correctly not assigned together, among all possible pairs. It is also adjusted for chance.

\section{Fowlkes-Mallows index}

It is a metric derived from the true positives (TP), false positives (FP), and false negatives (FN) as follows: $\mathrm{FMI}=\sqrt{\frac{\mathrm{TP}}{\mathrm{TP}+\mathrm{FP}} \cdot \frac{\mathrm{TP}}{\mathrm{TP}+\mathrm{FN}}}$

\section{Silhouette coefficient [29]}

It is a clustering metric derived by comparing the mean intra-cluster distance and the mean inter-cluster distance. 


\section{Differential expression analysis}

We perform the differential expression analysis using the scanpy package on the simulation as the groups are pre-defined. For each method, we extracted the differentially expressed genes for each cell group by performing a $t$ test of one group against the rest groups. We used Benjamini-Hochberg correction for multiple hypothesis testing to obtain adjusted $p$ value $\left(\mathrm{pval}_{\text {adj }}\right)$. Since each method has generated different differentially expressed genes, we extracted the top 500 differentially expressed genes for each group and pooled the differentially expressed genes for all of the groups. Using 1-pval ${ }_{\text {adj }}$ as the DE calling probability and the true differentially expressed genes (by Splatter) as the truth measure, we calculated the area under the curve (AUC) for the ROC curve for each method using the scikit-learn python package.

\section{RNA FISH validation}

We obtain a Drop-Seq dataset (GSE99330) and its RNA FISH dataset from a melanoma cell line, as described by Torre et al. [36]. The summary of the dataset is listed in Additional file 3: Table S1. For the comparison between RNA FISH and the corresponding Drop-Seq experiment, we keep genes with a variance over mean ratio $>0.5$, the same as other datasets in this study, leaving six genes in common between the FISH and the Drop-Seq datasets.

For GINI coefficient calculation, we first normalize the cells in each dataset using a housekeeping gene (glyceraldehyde 3-phosphate dehydrogenase, or GAPDH)-based factor, as done by others [20]. We remove GAPDH outlier cells (defined here as the cells below the 10th and above the 90th percentiles). Then, we rescale each data point by a GAPDH-based factor, as follows:

$$
\begin{aligned}
& \text { data }[\text { cell, gene }]=\text { data }[\text { cell, gene }] * \text { factor }(\text { cell }) \\
& \text { where factor }(\text { cell })=\operatorname{mean}(\text { data }[:, \text { GAPDH }]) / \text { data }[\text { cell, GAPDH }]
\end{aligned}
$$

Then, we compute GINI coefficient, as done in SAVER [20]. For distribution normalization, the procedure is the same except that we first normalize each gene by an efficiency factor (defined as the ratio between its mean value for FISH and its value for the imputation method). We calculate the MSEs and Pearson's coefficients with the following formulas:

$$
\begin{aligned}
& \operatorname{MSE}(\text { gene }, \text { method })=\sum_{\text {cell }}\left(X_{\mathrm{FISH}}(\text { gene }, \text { cell })-X_{\text {method }}(\text { gene }, \text { cell })\right)^{2} \\
& \operatorname{Corr}(\text { gene }, \text { method })=\frac{\operatorname{Cov}\left(X_{\mathrm{FISH}}(\text { gene }), X_{\text {method }}(\text { gene })\right)}{\operatorname{Var}\left(X_{\mathrm{FISH}}(\text { gene })\right) \cdot \operatorname{Var}\left(X_{\text {method }}(\text { gene })\right)}
\end{aligned}
$$

where $X$ is the input matrix of gene expression from RNA-FISH or Drop-Seq, Cov is the covariance, and Var is the variance.

\section{Supplementary information}

Supplementary information accompanies this paper at https://doi.org/10. 1186/s13059-019-1837-6.

Additional file 1. Explanatory figures for Deeplmpute's preprocessing and for the masking experiment.

Additional file 2. Dropout and activation function optimization experiments for Deeplmpute's architecture.

Additional file 3. Summary table of the dataset used in this paper.

\section{Peer review information}

Yixin Yao and Barbara Cheifet were the primary editors on this article and managed its editorial process and peer review in collaboration with the rest of the editorial team.

\section{Authors' contributions}

LG envisioned this project. CA implemented the project and conducted the analysis with the help from OP, BY, and XZ. CA, BY, and LG wrote the manuscript. All authors have read and agreed on the manuscript.

\section{Funding}

The authors thank Dr. Arjun Raj and Eduardo Torre for providing the data for RNA FISH and Drop-Seq. This research was supported by grants

K01ES025434 awarded by NIEHS through funds provided by the trans-NIH Big Data to Knowledge (BD2K) initiative (www.bd2k.nih.gov), P20 COBRE GM103457 awarded by NIH/NIGMS, R01 LM012373 awarded by NLM, and R01 HD084633 awarded by NICHD to L.X. Garmire.

\section{Availability of data and materials}

sCRNA-seq Datasets

In this study, we evaluate imputation metrics on nine datasets. Four of them (Jurkat, 293T, neuron9k, and Mouse1M) are downloaded from the 10X Genomics support website (https://support.10xgenomics.com/single-cellgene-expression/datasets). Briefly, the Jurkat dataset is extracted from the Jurkat cell line (human blood). 293T is a blood cell line derived from HEK293T that expresses a mutant version of the SV40 large T antigen. The neuron9k dataset contains brain cells from an E18 mouse. Mouse1M also contains brain cells from an E18 mouse. The FISH and GSE99330 data were both extracted from the same melanoma cell line WM989-A6 [36]. Two other datasets are taken from GSE67602 [52], composed of mouse interfollicular epidermis cells and the Hrvatin dataset GSE102827 [37] dataset, extracted from primary visual cortex of C57BL6/J mice. Additionally, we simulate a dataset with the Splatter package [14] with parameters dropout.shape = 0.5 , dropout.mid $=1,4000$ genes and 2000 cells split into 5 groups with proportions $10 \%, 10 \%, 20 \%, 20 \%$, and $40 \%$. Each gene in each group is automatically assigned a differential expression (DE) factor, where 1 is not differentially expressed, a value less than 1 is downregulated, and more than 1 is upregulated.

Third party software

For comparison, we use the latest version of SAVER (v1.1.1) at https://github. $\mathrm{com} /$ mohuangx/SAVER/releases, Sclmpute (v0.0.9) at https://github.com/ Vivianstats/sclmpute, Drlmpute (v1.0) available as a CRAN package, MAGIC (v1.4.0) at https://github.com/KrishnaswamyLab/magic, VIPER (v1.0) at https:// github.com/ChenMengjie/VIPER/releases, and DCA (0.2.2) at https://github. $\mathrm{com} /$ theislab/dca. We preprocess the datasets according to each method's standard: using a square root transformation for MAGIC, log transformation for Deeplmpute (with a pseudo count of 1), but raw counts for sclmpute, Drlmpute, SAVER, and DCA. For VIPER, we remove all genes with a null total count and rescale each cell to a library size of one million (RPM normalization) as recommended.

Deeplmpute's material

Deeplmpute package and its documentation are freely available on GitHubhttps://github.com/lanagarmire/Deeplmpute [53] under the MIT license. The software as well as the source code to reproduce the figures of this paper was deposited on Zenodo https://doi.org/10.5281/zenodo. 3459902 [54].

Ethics approval and consent to participate No ethnical approval was required for this study. 


\section{Competing interests}

The authors declare that they have no competing interests.

\section{Author details}

'Department of Information and Computer Science, University of Hawaii at Manoa, Honolulu, HI 96816, USA. ${ }^{2}$ Department of Epidemiology, University of Hawaii Cancer Center, 701 llalo Street, Honolulu, HI 96813, USA. ${ }^{3}$ Department of Molecular Biology and Bioengineering, University of Hawaii at Manoa, Honolulu, HI 96816, USA. ${ }^{4}$ Department of Computational Medicine and Bioinformatics, University of Michigan, Ann Arbor, Ml 48105, USA.

Received: 6 July 2019 Accepted: 26 September 2019

Published online: 18 October 2019

\section{References}

1. Usoskin D, Furlan A, Islam S, Abdo H, Lönnerberg P, Lou D, et al. Unbiased classification of sensory neuron types by large-scale single-cell RNA sequencing. Nat Neurosci. 2015;18:145 Nature Publishing Group.

2. Villani A-C, Satija R, Reynolds G, Sarkizova S, Shekhar K, Fletcher J, et al. Single-cell RNA-seq reveals new types of human blood dendritic cells, monocytes, and progenitors. Science. 2017;356:eaah4573 American Association for the Advancement of Science.

3. Zeisel A, Muñoz-Manchado AB, Codeluppi S, Lönnerberg P, La Manno G, Juréus $A$, et al. Cell types in the mouse cortex and hippocampus revealed by single-cell RNA-seq. Science. 2015;347:1138-42 American Association for the Advancement of Science.

4. Jaitin DA, Kenigsberg E, Keren-Shaul H, Elefant N, Paul F, Zaretsky I, et al. Massively parallel single-cell RNA-seq for marker-free decomposition of tissues into cell types. Science. 2014;343:776-9 American Association for the Advancement of Science.

5. Kriegstein A, Pollen AA, Nowakowski TJ, Shuga J, Wang X, Leyrat AA, et al. Low-coverage single-cell mRNA sequencing reveals cellular heterogeneity and activated signaling pathways in developing cerebral cortex. 2014;

6. Treutlein B, Brownfield DG, Wu AR, Neff NF, Mantalas GL, Espinoza FH, et al. Reconstructing lineage hierarchies of the distal lung epithelium using single-cell RNA-seq. Nature. 2014;509:371 Nature Publishing Group.

7. Tirosh I, Venteicher AS, Hebert C, Escalante LE, Patel AP, Yizhak K, et al. Single-cell RNA-seq supports a developmental hierarchy in human oligodendroglioma. Nature. 2016;539:309 Nature Publishing Group.

8. Shalek AK, Satija R, Adiconis X, Gertner RS, Gaublomme JT, Raychowdhury R, et al. Single-cell transcriptomics reveals bimodality in expression and splicing in immune cells. Nature. 2013;498:236 Nature Publishing Group.

9. Tang F, Barbacioru C, Bao S, Lee C, Nordman E, Wang X, et al. Tracing the derivation of embryonic stem cells from the inner cell mass by single-cell RNA-Seq analysis. Cell Stem Cell. 2010;6:468-78 Elsevier.

10. Kim JK, Kolodziejczyk AA, llicic T, Teichmann SA, Marioni JC. Characterizing noise structure in single-cell RNA-seq distinguishes genuine from technical stochastic allelic expression. Nat Commun. 2015;6:8687 Nature Publishing Group.

11. Kolodziejczyk AA, Kim JK, Svensson V, Marioni JC, Teichmann SA. The technology and biology of single-cell RNA sequencing. Mol Cell. 2015;58: 610-20 Elsevier.

12. Jia C, Hu Y, Kelly D, Kim J, Li M, Zhang NR. Accounting for technical noise in differential expression analysis of single-cell RNA sequencing data. Nucleic Acids Res. 2017:45:10978-88.

13. Andrews TS, Hemberg M. Modelling dropouts allows for unbiased identification of marker genes in scRNASeq experiments [Internet]. bioRxiv. 2016:065094 [cited 2019 Apr 26]. Available from: https://www.biorxiv.org/ content/early/2016/07/21/065094.

14. Zappia L, Phipson B, Oshlack A. Splatter: simulation of single-cell RNA sequencing data. Genome Biol. 2017;18:174 BioMed Central.

15. Zhu X, Ching T, Pan X, Weissman SM, Garmire L. Detecting heterogeneity in singlecell RNA-Seq data by non-negative matrix factorization. PeerJ. 2017;5:e2888.

16. Poirion O, Zhu X, Ching T, Garmire LX. Using single nucleotide variations in single-cell RNA-seq to identify subpopulations and genotype-phenotype linkage. Nat Commun. 2018;9:4892.

17. Zhu X, Wolfgruber TK, Tasato A, Arisdakessian C, Garmire DG, Garmire LX. Granatum: a graphical single-cell RNA-Seq analysis pipeline for genomics scientists. Genome Med. 2017:9:108 BioMed Central.

18. van Dijk D, Sharma R, Nainys J, Yim K, Kathail P, Carr AJ, et al. Recovering Gene Interactions from Single-Cell Data Using Data Diffusion. Cell. 2018;174: 716-29.e27
19. Li WW, Li JJ. An accurate and robust imputation method sclmpute for single-cell RNA-seq data. Nat Commun. 2018;9:997.

20. Huang M, Wang J, Torre E, Dueck H, Shaffer S, Bonasio R, et al. SAVER: gene expression recovery for single-cell RNA sequencing. Nat Methods. 2018;15: 539-42.

21. Gong W, Kwak I-Y, Pota P, Koyano-Nakagawa N, Garry DJ. Drlmpute: imputing dropout events in single cell RNA sequencing data. BMC Bioinformatics. 2018;19:220.

22. Chen M, Zhou X. VIPER: variability-preserving imputation for accurate gene expression recovery in single-cell RNA sequencing studies [internet]. Genome Biol. 2018; Available from: https://doi.org/10.1186/s13059-018-1575-1.

23. Eraslan G, Simon LM, Mircea M, Mueller NS, Theis FJ. Single-cell RNA-seq denoising using a deep count autoencoder. Nat Commun. 2019;10:390.

24. Lin P, Troup M, Ho JWK. CIDR: ultrafast and accurate clustering through imputation for single-cell RNA-seq data. Genome Biol. 2017;18:59.

25. Ronen J, Akalin A. netSmooth: network-smoothing based imputation for single cell RNA-seq. F1000Res. 2018;7:8

26. Zhang $L$, Zhang S. Comparison of computational methods for imputing singlecell RNA-sequencing data. IEEE/ACM transactions on computational biology and bioinformatics. 2018. https://doi.org/10.1109/TCBB.2018.2848633.

27. Ching T, Zhu X, Garmire LX. Cox-nnet: an artificial neural network method for prognosis prediction of high-throughput omics data. PLoS Comput Biol. 2018;14:e1006076.

28. Alakwaa FM, Chaudhary K, Garmire LX. Deep learning accurately predicts estrogen receptor status in breast cancer metabolomics data. J Proteome Res. 2018;17:337-47.

29. Chaudhary K, Poirion OB, Lu L, Garmire LX. Deep learning-based multiomics integration robustly predicts survival in liver cancer. Clin Cancer Res. 2018;24:1248-59.

30. Ching T, Himmelstein DS, Beaulieu-Jones BK, Kalinin AA, Do BT, Way GP, et al. Opportunities and obstacles for deep learning in biology and medicine. J R Soc Interface. 2018;15 Available from: https://doi.org/10.1098/ rsif.2017.0387.

31. Tan J, Doing G, Lewis KA, Price CE, Chen KM, Cady KC, et al. Unsupervised extraction of stable expression signatures from public compendia with an ensemble of neural networks. Cell Syst. 2017;5:63-71.e6.

32. Beaulieu-Jones BK, Greene CS, Pooled Resource Open-Access ALS Clinical Trials Consortium. Semi-supervised learning of the electronic health record for phenotype stratification. J Biomed Inform. 2016;64:168-78.

33. Beaulieu-Jones BK, Moore JH. Missing data imputation in the electronic health record using deeply learned autoencoders. Pac Symp Biocomput. 2017;22:207-18

34. Chiang C-C, Fu H-C. A divide-and-conquer methodology for modular supervised neural network design. Neural Networks, 1994 IEEE World Congress on Computational Intelligence, 1994 IEEE International Conference on. 1994. p. 119-124 vol.1.

35. Garmire LX, Subramaniam S. Evaluation of normalization methods in mammalian microRNA-Seq data. RNA. 2012;18:1279-88.

36. Torre E, Dueck H, Shaffer S, Gospocic J, Gupte R, Bonasio R, et al. Rare cell detection by single-cell RNA sequencing as guided by single-molecule RNA FISH. Cell Syst. 2018;6:171-9 Elsevier.

37. Hrvatin S, Hochbaum DR, Nagy MA, Cicconet M, Robertson K, Cheadle L, et al. Single-cell analysis of experience-dependent transcriptomic states in the mouse visual cortex. Nat Neurosci. 2018;21:120-9 nature.com.

38. McInnes L, Healy J, Melville J. UMAP: uniform manifold approximation and projection for dimension reduction [Internet]. arXiv [stat.ML]. 2018; Available from: http://arxiv.org/abs/1802.03426.

39. Traag $\vee$, Waltman L, van Eck NJ. From Louvain to Leiden: guaranteeing wellconnected communities [Internet]. arXiv [cs.SI]. 2018; Available from: http:// arxiv.org/abs/1810.08473.

40. Blondel VD, Guillaume J-L, Lambiotte R, Lefebvre E. Fast unfolding of communities in large networks. J Stat Mech. 2008;2008:P10008 IOP Publishing.

41. Jouppi NP, Young C, Patil N, Patterson D, Agrawal G, Bajwa R, et al. Indatacenter performance analysis of a tensor processing unit. In: Proceedings of the 44th Annual International Symposium on Computer Architecture. New York: ACM; 2017. p. 1-12. https://arxiv.org/abs/1704.04760.

42. Shanahan J, Dai L. Large scale distributed data science from scratch using Apache Spark 2.0. In: Proceedings of the 26th International Conference on World Wide Web Companion. Republic and Canton of Geneva: International World Wide Web Conferences Steering Committee; 2017. p. 955-7. 
43. Mehta P, Dorkenwald S, Zhao D, Kaftan T, Cheung A, Balazinska M, et al. Comparative evaluation of big-data systems on scientific image analytics workloads. Proc VLDB Endowment. 2017;10:1226-37 VLDB Endowment.

44. Tian T, Wan J, Song Q, Wei Z. Clustering single-cell RNA-seq data with a model-based deep learning approach [Internet]. Nat Mach Intell. 2019:191-8 Available from: https://doi.org/10.1038/s42256-019-0037-0.

45. Chollet F. Keras. 2015; Available from: https:/scholar.google.ca/scholar?cluster= $17868569268188187229,14781281269997523089,11592651756311359484,6655$ $887363479483357,415266154430075794,6698792910889103855,69419872326$ $7881416,11861311255053948243,5629189521449088544,107014270213879202$ $84,14698280927700770473 \&$ hl=en\&as_sdt $=0,5 \&$ sciodt $=0,5$.

46. Abadi M, Barham P, Chen J, Chen Z, Davis A, Dean J, et al. TensorFlow: a system for large-scale machine learning. In Proceedings of the 12th USENIX Symposium on Operating Systems Design and Implementation (OSDI 16), USENIX Association. 2016. p. 265-83.

47. Satija R, Farrell JA, Gennert D, Schier AF, Regev A. Spatial reconstruction of single-cell gene expression data. Nat Biotechnol. 2015;33:495-502 nature.com.

48. Wolf FA, Angerer P, Theis FJ. SCANPY: large-scale single-cell gene expression data analysis. Genome Biol. 2018;19:15 genomebiology.biomedcentral.com.

49. Srivastava N, Hinton G, Krizhevsky A, Sutskever I, Salakhutdinov R. Dropout: a simple way to prevent neural networks from overfitting. J Mach Learn Res. 2014;15:1929-58 JMLR. org.

50. Kingma DP, Ba J. Adam: a method for stochastic optimization. arXiv preprint arXiv:1412 6980. 2014;

51. Ruder S. An overview of gradient descent optimization algorithms. arXiv preprint arXiv:1609 04747. 2016

52. Joost S, Zeisel A, Jacob T, Sun X, La Manno G, Lönnerberg P, et al. Singlecell transcriptomics reveals that differentiation and spatial signatures shape epidermal and hair follicle heterogeneity. Cell Syst. 2016;3:221-37.e9.

53. Arisdakessian C, Poirion O, Yunits B, Zhu X, Garmire LX. Deeplmpute: an accurate, fast and scalable deep neural network method to impute singlecell RNA-Seq data. Github. 2019. https://github.com/lanagarmire/ Deeplmpute.

54. Arisdakessian C, Poirion O, Yunits B, Zhu X, Garmire LX. Deeplmpute: an accurate, fast and scalable deep neural network method to impute singlecell RNA-Seq data. Zenodo. 2019. https://doi.org/10.5281/zenodo.3459902.

\section{Publisher's Note}

Springer Nature remains neutral with regard to jurisdictional claims in published maps and institutional affiliations.

Ready to submit your research? Choose BMC and benefit from:

- fast, convenient online submission

- thorough peer review by experienced researchers in your field

- rapid publication on acceptance

- support for research data, including large and complex data types

- gold Open Access which fosters wider collaboration and increased citations

- maximum visibility for your research: over $100 \mathrm{M}$ website views per year

At $\mathrm{BMC}$, research is always in progress.

Learn more biomedcentral.com/submissions 